\title{
TWISTED HODGE FILTRATION: CURVATURE OF THE DETERMINANT
}

\author{
PHILIPP NAUMANN
}

\begin{abstract}
Given a holomorphic family $f: \mathcal{X} \rightarrow S$ of compact complex manifolds and a relative ample line bundle $L \rightarrow \mathcal{X}$, the higher direct images $R^{n-p} f_{*} \Omega_{\mathcal{X} / S}^{p}(L)$ carry a natural hermitian metric. An explicit formula for the curvature tensor of these direct images is given in Na16. We prove that the determinant of the twisted Hodge filtration $F_{L}^{p}=\oplus_{i \geq p} R^{n-i} \Omega_{\mathcal{X} / S}^{i}(L)$ is (semi-) positive on the base $S$ if $L$ itself is (semi-) positive on $\mathcal{X}$.
\end{abstract}

\section{INTRODUCTION}

For an ample line bundle $L$ on a compact complex manifolds $X$ of dimension $n$, the cohomology groups $H^{n-p}\left(X, \Omega_{X}^{p}(L)\right)$ are critical with respect to Kodaira-Nakano vanishing. More generally, we consider the higher direct image sheaves $R^{n-p} f_{*} \Omega_{\mathcal{X} / S}^{p}(L)$ for a proper holomorphic submersion $f: \mathcal{X} \rightarrow S$ of complex manifolds and a line bundle $L \rightarrow \mathcal{X}$, which is positive along the fibers $X_{s}=f^{-1}(s)$. The understanding of this situation has applications to moduli problems. An explicit curvature formula for these higher direct images is given in [Na16]. In general, the curvature of the intermediate higher direct images contains positive and negative contributions. Therefore, we consider the twisted Hodge filtration

$$
F_{L}^{p}:=\oplus_{i \geq p} R^{n-i} \Omega_{\mathcal{X} / S}^{i}(L)
$$

This allows us to offset the negative terms by the positive ones. The result is that $\operatorname{det}\left(F_{L}^{p}\right)$ is (semi-) positive on the base $S$ if $L$ is (semi-) positive and relatively ample on $\mathcal{X}$. This idea comes from [Sch16].

\section{Differential geometric Setup And proof of the Result}

First we recall the setting from Na16. Let $f: \mathcal{X} \rightarrow S$ be a proper holomorphic submersion and $(L, h)$ a line bundle on $\mathcal{X}$. The curvature form of the hermitian line bundle is given by

$$
\omega_{\mathcal{X}}:=2 \pi \cdot c_{1}(L, h)=-\sqrt{-1} \partial \bar{\partial} \log h .
$$

We consider the case where the hermitian bundle $(L, h)$ is relative ample, which means that

$$
\omega_{X_{s}}:=\left.\omega\right|_{X_{s}}
$$

are Kähler forms on the $n$-dimensional fibers $X_{s}$. Then one has the notion of a horizontal lift $v_{s}$ of a tangent vector $\partial_{s}$ on the base $S$ and we get a representative of the Kodaira-Spencer class

$$
A_{s}:=\left.\bar{\partial}\left(v_{s}\right)\right|_{X_{s}} .
$$

Note that we have $L_{v_{s}}\left(\omega_{X_{s}}\right)^{n}=0$ (see [Be11]). Furthermore, one sets

$$
\varphi:=\left\langle v_{s}, v_{s}\right\rangle_{\omega_{\mathcal{X}}}
$$

which is called the geodesic curvature. The coherent sheaf $R^{n-p} f_{*} \Omega_{\mathcal{X} / S}^{p}(L)$ is locally free on $S$ outside a proper subvariety. In the case $n=p$ and $L$ ample, the sheaf $f_{*}\left(K_{\mathcal{X} / S} \otimes L\right)$ is locally free by the Ohsawa-Takegoshi extension theorem (see [Be09]). We assume the local freeness of

$$
R^{n-p} f_{*} \Omega_{\mathcal{X} / S}^{p}(L)
$$

2000 Mathematics Subject Classification. 32L10, 32G05, 14Dxx.

Key words and phrases. Curvature of higher direct image sheaves, Deformations of complex structures, Families, Fibrations, Twisted Hodge filtration. 
in the general case, hence the statement of the Grothendieck-Grauert comparison theorem holds. Now Lemma 2 of Sch12] applies, which says that we can represent local sections of $R^{n-p} f_{*} \Omega_{\mathcal{X} / S}^{p}(L)$ by $\bar{\partial}$ closed $(p, n-p)$-forms on the total space, whose restrictions to the fibers are harmonic. Let $\left\{\psi^{1}, \ldots, \psi^{r}\right\}$ be a local frame of the direct image consisting of such sections around a fixed point $s \in S$. We denote by $\left\{\left(\partial / \partial s_{i}\right) \mid i=1, \ldots, \operatorname{dim} S\right\}$ a basis of the complex tangent space $T_{s} S$ of $S$ over $\mathbb{C}$, where $s_{i}$ are local holomorphic coordinates on $S$. The natural inner product is given by

$$
\left(\psi^{k}, \psi^{l}\right)=\int_{X} \psi_{A_{p} \bar{B}_{q}}^{k} \psi_{C_{q} \bar{D}_{p}}^{\bar{l}} g^{\bar{D}_{p} A_{p}} g^{\bar{B}_{q} C_{q}} h g d V,
$$

Let $A_{i \bar{\beta}}^{\alpha}(z, s) \partial_{\alpha} d z^{\bar{\beta}}=\bar{\partial}\left(v_{i}\right)_{X_{s}}$ be the $\bar{\partial}$-closed representative of the Kodaira-Spencer class of $\partial_{i}$ described above. Then the cup product together with contraction defines

$$
\begin{gathered}
A_{i \bar{\beta}}^{\alpha} \partial_{\alpha} d z^{\bar{\beta}} \cup: \mathcal{A}^{0, n-p}\left(X_{s}, \Omega_{X_{s}}^{p}\left(\left.L\right|_{X_{s}}\right)\right) \rightarrow \mathcal{A}^{0, n-p+1}\left(X_{s}, \Omega_{X_{s}}^{p-1}\left(\left.L\right|_{X_{s}}\right)\right) \\
A_{\bar{\jmath} \alpha}^{\bar{\beta}} \partial_{\bar{\beta}} d z^{\alpha} \cup: \mathcal{A}^{0, n-p}\left(X_{s}, \Omega_{X_{s}}^{p}\left(\left.L\right|_{X_{s}}\right)\right) \rightarrow \mathcal{A}^{0, n-p-1}\left(X_{s}, \Omega_{X_{s}}^{p+1}\left(L_{X_{s}}\right)\right)
\end{gathered}
$$

where $p>0$ in (11) and $p<n$ in (2). Note that this is a formal analogy to the derivative of the period map in the classical case (see Gr70]). We will apply the above cup products to harmonic $(p, n-p)$-forms. In general, the results are not harmonic.

When applying the Laplace operator to $(p, q)$-forms with values in $L$ on the fibers $X_{s}$, we have

$$
\square_{\partial}-\square_{\bar{\partial}}=(n-p-q) \cdot \mathrm{id}
$$

due to the definition $\omega_{X_{s}}=\left.\omega_{\mathcal{X}}\right|_{X_{s}}$ and the Bochner-Kodaira-Nakano identity. Thus, we write $\square=\square \partial=$ $\square_{\bar{\partial}}$ in the case $q=n-p$. By considering an eigenfunction decomposition and using the identity (3), we obtain that all eigenvalues of $\square$ are 0 or greater than 1 , hence the operator $(\square-1)^{-1}$ exists. We use the notation $\psi^{\bar{l}}:=\overline{\psi^{l}}$ for sections $\psi^{l}$ and write $g d V=\omega_{X_{s}} / n$ !. The result from [Na16] is

Theorem 1. Let $f: \mathcal{X} \rightarrow S$ be a proper holomorphic submersion and $(L, h) \rightarrow \mathcal{X}$ a relative ample line bundle. With the objects described above, the curvature of $R^{n-p} f_{*} \Omega_{\mathcal{X} / S}^{p}(L)$ is given by

$$
\begin{aligned}
R_{\bar{\imath} \bar{\jmath}}^{\bar{l} k}(s)= & \int_{X_{s}} \varphi_{i \bar{\jmath}} \cdot\left(\psi^{k} \cdot \psi^{\bar{l}}\right) g d V \\
& +\int_{X_{s}}(\square+1)^{-1}\left(A_{i} \cup \psi^{k}\right) \cdot\left(A_{\bar{\jmath}} \cup \psi^{\bar{l}}\right) g d V \\
& +\int_{X_{s}}(\square-1)^{-1}\left(A_{i} \cup \psi^{\bar{l}}\right) \cdot\left(A_{\bar{\jmath}} \cup \psi^{k}\right) g d V
\end{aligned}
$$

If $L \rightarrow \mathcal{X}$ is ample, the only contribution, which may be negative, originates from the harmonic parts in the third term

$$
-\int_{X_{s}} H\left(A_{I} \cup \psi^{\bar{l}}\right) \overline{H\left(A_{j} \cup \psi^{\bar{k}}\right)} g d V .
$$

From now on, we assume that the base space $S$ is one dimensional. Let $\partial_{s}$ be a tangent vector and $A=A_{s}$ the corresponding $\bar{\partial}$-closed Dolbeault representative of the Kodaira-Spencer class. By considering an eigenvector decomposition, we have the following estimate for the curvature of $R^{n-p} \Omega_{\mathcal{X} / S}^{p}(L)$ for a semi-positive, relatively ample line bundle $L$ (see also [Sch16, Corollary 5]):

\section{Corollary 1.}

$$
R(A, \bar{A}, \psi, \bar{\psi}) \geq\|H(A \cup \psi)\|^{2}-\|H(\bar{A} \cup \psi)\|^{2}
$$

Now we formalize the situation: The cup products (11) and (2) give rise to cup products for cohomology classes ( $\psi$ is assumed to be harmonic).

$$
\begin{array}{cll}
A_{p}: H^{n-p}\left(X_{s}, \Omega_{X_{s}}^{p}\left(\left.L\right|_{X_{s}}\right)\right) \rightarrow H^{n-p+1}\left(X_{s}, \Omega_{X_{s}}^{p-1}\left(\left.L\right|_{X_{s}}\right)\right) & ; \quad[\psi] \mapsto[H(A \cup \psi)] \\
\bar{A}_{p}: H^{n-p}\left(X_{s}, \Omega_{X_{s}}^{p}\left(\left.L\right|_{X_{s}}\right)\right) \rightarrow H^{n-p-1}\left(X_{s}, \Omega_{X_{s}}^{p+1}\left(L_{X_{s}}\right)\right) & ; \quad[\psi] \mapsto[H(\bar{A} \cup \psi)]
\end{array}
$$

We have ([Sch16, Lemma 5]) 
Lemma 1. $\bar{A}_{p}=\left(A_{p+1}\right)^{*}$, i.e. the map $\bar{A}_{p}$ is the adjoint of the map $A_{p+1}$.

Proof. For any harmonic $\chi \in \mathcal{A}^{(p, n-p)}\left(X_{s},\left.L\right|_{X_{s}}\right)$ we have $(H(\bar{A} \cup \psi), \chi)=(\bar{A} \cup \psi, \chi)=(\psi, A \cup \chi)=$ $(\psi, H(A \cup \chi))$.

In the spirit of the Hodge filtration we define for $0 \leq p \leq n$

\section{Definition 1.}

$$
F_{L}^{p}:=\bigoplus_{i \geq p} R^{n-i} \Omega_{\mathcal{X} / S}^{i}(L)
$$

Then we have

Theorem 2. The line bundle $\operatorname{det}\left(F_{L}^{p}\right)$ is (semi-) positive on $S$, if $L$ is (semi-) positive and relatively ample on $\mathcal{X}$.

Proof. We choose a local holomorphic frame $\left\{\psi^{\alpha}\right\} \subset \mathcal{A}^{(p, n-p)}\left(X_{s},\left.L\right|_{X_{s}}\right)$ of harmonic forms and compute

$$
\begin{aligned}
\sum_{\alpha}\left\|H\left(A \cup \psi^{\alpha}\right)\right\|^{2} & =\sum\left(H\left(A \cup \psi^{\alpha}\right), H\left(A \cup \psi^{\alpha}\right)\right) \\
& =\sum\left(H\left(\bar{A} \cup A \cup \psi^{\alpha}\right), \psi^{\alpha}\right) \\
& =\sum\left(\left(A_{p}^{*} \circ A_{p}\right) \psi^{\alpha}, \psi^{\alpha}\right) \\
& =\operatorname{tr}\left(A_{p}^{*} A_{p}\right)
\end{aligned}
$$

Analogously

$$
\begin{aligned}
\sum_{\alpha}\left\|H\left(\bar{A} \cup \psi^{\alpha}\right)\right\|^{2} & =\sum\left(H\left(\bar{A} \cup \psi^{\alpha}\right), H\left(\bar{A} \cup \psi^{\alpha}\right)\right) \\
& =\sum\left(H\left(A \cup \bar{A} \cup \psi^{\alpha}\right), \psi^{\alpha}\right) \\
& =\sum\left(\left(\bar{A}_{p}^{*} \circ \bar{A}_{p}\right) \psi^{\alpha}, \psi^{\alpha}\right) \\
& =\operatorname{tr}\left(\bar{A}_{p}^{*} \bar{A}_{p}\right) \\
& =\operatorname{tr}\left(A_{p+1} A_{p+1}^{*}\right),
\end{aligned}
$$

because of Lemma 1. Note that we have $\operatorname{tr}\left(A_{p}^{*} A_{p}\right)=\operatorname{tr}\left(A_{p} A_{p}^{*}\right)$. Now the result follows from Corollary 1 , since the curvature of the determinant of a bundle is the trace of the curvature form of the bundle.

\section{REFERENCES}

[Be09] B. Berndtsson: Curvature of vector bundles associated to holomorphic fibrations, Ann. Math. 169, 531-560 (2009).

[Be11] B. Berndtsson: Strict and nonstrict positivity of direct image bundles, Math. Zeitschrift, Vol. 269, 1201-1218 (2011).

[De12] J.-P. Demailly: Complex Analytic and Differential Geometry, https://www-fourier.ujf-grenoble.fr/ demailly/manuscripts/agbook.pdf, Grenoble (2012).

[Gr70] P.A. Griffiths: Periods of integrals on algebraic manifolds III: Some differential-geometric properties of the period mapping, Publ. Math. IHES 38, 125-180 (1970).

[MT08] C. Mourougane, S. Takayama: Hodge metrics and the curvature of higher direct images, Ann. Sci. Ec. Norm. Supér. (4) 41, 905-924 (2008).

[Na16] P. Naumann: Curvature of higher direct images, arXiv:1611.09117 (2016).

[Sch93] G. Schumacher: The curvature of the Petersson-Weil metric on the moduli space of Kähler-Einstein manifolds, Ancona, V. (ed.) et al., Complex analysis and geometry. New York: Plenum Press. The University Series in Math., 339-354 (1993).

[Sch12] G. Schumacher: Positivity of relative canonical bundles and applications, Invent. Math. 190, 1-56 (2012).

[Sch13] G. Schumacher: Erratum to: Positivity of relative canonical bundles and applications, Invent. Math. 192, 253-255 (2013).

[Sch16] G. Schumacher: Moduli of canonically polarized manifolds, higher order Kodaira-Spencer maps, and an analogy to Calabi-Yau manifolds, to appear in (2016). 
PHILIPP NAUMANN

Fachbereich Mathematik und Informatik, Philipps-Universität Marburg, Lahnberge, Hans-Meerwein-Strasse, D-35032 Marburg,Germany

E-mail address: naumann@mathematik.uni-marburg.de 